\title{
UTILIZAÇÃO DO DIÓXIDO DE CARBONO SUPERCRÍTICO NA CONCENTRAÇÃO DE TOCOFERÓIS DO DESTILADO DESODORIZADO DO ÓLEO DE SOJA ${ }^{1}$
}

\author{
JÚLIO MARIA DE ANDRADE ARAUJO ${ }^{2}$, ANA PAULA NOGUEIRA NICOLINO ${ }^{3}$ e CELSO BLATT ${ }^{4}$
}

\begin{abstract}
RESUMO - O objetivo deste trabalho foi examinar a possibilidade do uso do dióxido de carbono supercrítico na extração e concentração de tocoferóis a partir do destilado desodorizado do óleo de soja. Trata-se da combinação seqüencial de duas extrações: pré-extração à temperatura de $80^{\circ} \mathrm{C}$ e pressão de 76 bars para remoção de substâncias interferentes e extração dos tocoferóis a $50^{\circ} \mathrm{C}$ e 197 bars. $\mathrm{O}$ coletor utilizado foi o octadesil-sílica, lavado com acetonitrila na pré-extração e hexano na extração, e a análise dos extratos obtidos foi feita por cromatografia de fase gasosa em coluna capilar. O resultado mostrou que o desodorizado do óleo de soja, contendo inicialmente 9,2\% de tocoferóis totais, pôde ser concentrado para 40,6\%. Sob as condições otimizadas, a extração pode ser realizada em 37 minutos.
\end{abstract}

Termos para indexação: extração supercrítica, cromatografia de fase gasosa.

\author{
UTILIZATION OF SUPERCRITICAL CARBON DIOXIDE FOR CONCENTRATION \\ OF TOCOPHEROLS FROM SOYBEAN OIL DEODORIZER DISTILLATE
}

\begin{abstract}
The aim of this work was to test the feasibility of the use of supercritical carbon dioxid for extraction and concentration of tocopherols from soybean sludge at temperatures of $80^{\circ} \mathrm{C}$ and pressures of 76 bars to remove interfereres during pre-extraction, and $50^{\circ} \mathrm{C}$ at 197 bars to extract tocopherols. An octadecyl-silici trap, washed with acetonitrile after pre-extraction following by hexane after extraction was used. The analysis of extracts was checked by capillary gas chromatography. The results showed that soybean sludge initially containing about $9.2 \%$ of tocopherols could be enriched to about $40.6 \%$. Under optimized conditions the extraction was performed in 37 minutes. The content of tocopherols in the extracts collected was checked by capillary gas chromatography.
\end{abstract}

Index terms: soybean sludge, supercritical extraction, gas chromatography.

\section{INTRODUÇ̃̃O}

Os tocoferóis são compostos lipossolúveis que possuem atividade de vitamina E. A síntese do $\alpha$-tocoferol foi descrita por Karrer et al. (1938). O produto sintético apresenta dois isômeros óticos, $\mathrm{d}$ e l-tocoferol, enquanto o produto natural possui somente a forma d. Os tocoferóis $(\alpha, \beta, \gamma$ e $\delta$-) têm diversas atividades biológicas ainda não totalmente

\footnotetext{
${ }^{1}$ Aceito para publicação em 14 de abril de 1999.

${ }^{2}$ Eng. Agrôn., Ph.D., Dep. de Tecnologia de Alimentos, Universidade Federal de Viçosa (UFV), CEP 36571-000 Viçosa, MG. E-mail: jaraujo@mail.ufv.br

${ }^{3}$ Eng. Alimentos, M.Sc., UFV.

${ }^{4}$ Quím., D.Sc., HP-Brasil, Av. Araunã no 125, CEP 06460-010 Barueri, São Paulo.
}

esclarecidas como prevenção da impotência sexual e de antioxidante natural.

Uma das fontes de vitamina E, com grande potencial de utilização industrial, são os resíduos da indústria de óleo de soja. O destilado obtido da desodorização desse óleo é composto principalmente por material orgânico volátil, que consiste em uma mistura complexa de ácidos graxos, glicerídeos (mono-, di- e triglicerídeos), aldeídos insaturados, hidrocarbonetos, pigmentos, esteróis, tocoferóis e água, além de produtos oxidados não identificados. Segundo Woerfel (1981), os tocoferóis perfazem em torno de $9 \%$ a $15 \%$ do peso total do resíduo .

O método tradicional utilizado em escala comercial na concentração de tocoferóis, após a remoção de esteróis via recristalização em álcool, é a destilação molecular. A técnica consiste em passar a amos- 
tra através de um trocador de calor num sistema de alto vácuo. A destilação geralmente é efetuada à temperatura próxima de $200-210^{\circ} \mathrm{C}$ e pressão de $0,001 \mathrm{~mm} \mathrm{Hg}$ por determinado período de tempo (Quaife \& Harris, 1946; Hickman, 1947). Entretanto, esse processo requer grandes quantidades de solventes orgânicos, diversos estádios para recuperação destes, e elevado consumo de energia.

Um método alternativo que vem sendo avaliado (Saito et al., 1989; Lee et al., 1991; Nicolino, 1995) para a produção de concentrado de tocoferóis é a extração com fluido supercrítico (EFS), com vantagens em relação ao processo de separação convencional, como extração líquido-líquido, destilação e adsorção. A principal vantagem da EFS é a fácil separação do solvente e material extraído isento de resíduos orgânicos. Além disso, os fluidos supercríticos oferecem maior e mais rápida transferência de massa, do que os processos tradicionais de separação.

O objetivo deste trabalho foi examinar a possibilidade do uso do dióxido de carbono supercrítico sob várias condições de extração, na concentração de tocoferóis, a partir do destilado desodorizado do óleo de soja (DDOS).

\section{MATERIAL E MÉTODOS}

O DDOS contendo $9,2 \%$ de tocoferóis totais foi doado pela indústria de óleo Olvebra Industrial S.A., Eldorado do Sul, RS. Esse destilado foi acondicionado num recipiente de polietileno, protegido de luz e calor, prevenindose, assim, sua oxidação.

A solução-padrão para quantificação do $\alpha$-tocoferol é formada por d,1- $\alpha$-tocoferol Merck ( $1 \mathrm{mg}$ ) dissolvido em hexano (grau cromatográfico) (1 mL). Dessa solução prepararam-se diluições para concentrações de 250, 125, 62,5 e 31,25 $\mu \mathrm{g}$ do padrão, as quais foram injetadas numa mesma quantidade $(1,6 \mu \mathrm{L})$ no cromatógrafo de fase gasosa.

A solução-padrão utilizada na otimização dos parâmetros da extração supercrítica dos tocoferóis foi: padrão Merck, contendo os quatro isômeros $(\alpha-, \beta-, \gamma$ - e $\delta$-tocoferol), numa concentração de $1 \mathrm{mg} / \mathrm{mL}$ de hexano (grau cromatográfico): $50 \mu \mathrm{L}$ da solução foram adsorvidos em papel-filtro.

Foi utilizado o cromatógrafo Hewllett Packard, modelo 5890, série II, com detector de ionização de chama equipado com sistema de injeção split-splitless em coluna capilar de sílica fundida (fase estacionária Metil-Silicone, equi- valente a SE-30, de $25 \mathrm{~m}$ de comprimento, 0,2 $\mathrm{mm}$ de diâmetro interno e espessura do filme de $0,33 \mu \mathrm{m})$. As condições de análise segundo Slover et al. (1983) e Marks (1988) foram: temperatura do injetor: $250^{\circ} \mathrm{C}$; temperatura da coluna: $200^{\circ} \mathrm{C}$ por $1 \mathrm{~min}$ e, então, programada na razão de $20^{\circ} \mathrm{C} / \mathrm{min}$ até a temperatura máxima de $300^{\circ} \mathrm{C}$, permanecendo isotérmica por $11 \mathrm{~min}$; temperatura do detector: $300^{\circ} \mathrm{C}$. O gás de arraste utilizado foi o hidrogênio, a uma vazão de $0,6 \mathrm{~mL} / \mathrm{min}$ e velocidade de $33,7 \mathrm{~cm} / \mathrm{s}$; para a chama, usaram-se o hidrogênio e o ar sintético. Utilizou-se injeção do tipo Split com fluxo do septo da purga de $3,7 \mathrm{~mL} / \mathrm{min}$ e pressão de 13,2 psi.

$\mathrm{O}$ equipamento básico utilizado neste trabalho foi $\mathrm{o}$ extrator de fluido supercrítico Hewlett Packard, modelo $7680 \mathrm{~A}$, equipado com restritor variável, permitindo o controle independente de fluxo e pressão. Os componentes extraídos são depositados no coletor empacotado com octadesil-sílica (ODS), localizado na saída do restritor, e lavados com solventes adequados (grau cromatográfico) para frascos de 2,0 mL.

A amostra $(20 \mu \mathrm{L}$ de DDOS) foi adsorvida em papelfiltro e transferida para o cartucho de extração de 7,0 mL. O procedimento de extração consistiu inicialmente de uma extração estática, seguida pela extração dinâmica à temperatura e pressão apresentadas na Tabela 1. A quantificação dos tocoferóis foi realizada utilizando-se a cromatografia de fase gasosa.

\section{RESULTADOS E DISCUSSÃO}

A extração de produtos naturais por meio do dióxido de carbono supercrítico $\left(\mathrm{CO}_{2}\right.$-SC) requer otimização das condições de extração e a caracterização do produto obtido. Neste estudo, a eficiência na extração dos tocoferóis foi medida à temperatura de 40,50 e $60^{\circ} \mathrm{C}$ e pressão variando de $110 \mathrm{a}$ 318 bars (Tabela 1). A extração de tocoferóis do DDOS, após a definição das condições otimizadas (Tabela 2), procedeu-se de forma rápida, e o extrato resultante foi utilizado diretamente na análise por cromatografia de fase gasosa.

Dados iniciais do destilado bruto (Fig. 1) sugerem que a maioria das substâncias detectadas são ácidos graxos livres (Gonçalves et al., 1991; Lee et al., 1991), os quais são mais solúveis que os monoglicerídeos, tocoferóis e diglicerídeos no $\mathrm{CO}_{2}$-SC, o que foi confirmado retirando-se uma alíquota e promovendo a metilação, sendo aquela 
analisada por cromatografia gasosa (CG). Portanto, a concentração de tocoferóis utilizando o $\mathrm{CO}_{2}-\mathrm{SC}$ sem nenhuma preparação inicial da amostra requer uma pré-extração em condições tais que promova a remoção de interferentes com propriedades de solubilidade similares às dos tocoferóis. Assim, avaliouse a possibilidade de uma pré-extração, visando à remoção de impurezas da amostra, o que permitiria maior concentração de tocoferóis na fase de extração.
Entre todas as temperaturas e densidades testadas na fase de pré-extração, a combinação que promoveu a maior remoção de interferentes foi $80^{\circ} \mathrm{C}$ e $0,15 \mathrm{~g} / \mathrm{mL}$ (76 bars) durante oito minutos, com fluxo de $3 \mathrm{~mL} / \mathrm{min}$.; e, dos solventes testados na lavagem do coletor mantido a $20^{\circ} \mathrm{C}$, a acetonitrila permitiu a eliminação de mais compostos, sem a remoção dos tocoferóis (Fig. 1).

Mudanças na temperatura e pressão foram utilizadas para controlar a densidade do fluido

TABELA 1. Condições testadas na otimização dos parâmetros para extração de tocoferóis.

\begin{tabular}{|c|c|c|}
\hline \multicolumn{3}{|c|}{ Pré-extração ${ }^{1}$} \\
\hline \multirow{2}{*}{ Fluxo do $\mathrm{CO}_{2}-\mathrm{SC}(\mathrm{mL} / \mathrm{min})$} & \multicolumn{2}{|c|}{ Tempo de extração (min) } \\
\hline & Estática & Dinâmica \\
\hline 0,5 & 0 & 5 \\
\hline 1,0 & 5 & 8 \\
\hline \multicolumn{3}{|l|}{3,0} \\
\hline Temperatura $\left({ }^{\circ} \mathrm{C}\right)$ & Pressão (bars) & Densidade $(\mathrm{g} / \mathrm{mL})$ \\
\hline 40 & 77 & 0,25 \\
\hline 50 & 85 & 0,25 \\
\hline 60 & 82 & 0,20 \\
\hline 70 & 88 & 0,20 \\
\hline 80 & 93 & 0,20 \\
\hline 80 & 76 & 0,15 \\
\hline \multicolumn{3}{|c|}{ Extração $^{2}$} \\
\hline \multirow{2}{*}{ Fluxo do $\mathrm{CO}_{2}-\mathrm{SC}(\mathrm{mL} / \mathrm{min})$} & \multicolumn{2}{|c|}{ Tempo de extração (min) } \\
\hline & Estática & Dinâmica \\
\hline 2 & 5 & 15 \\
\hline 3 & 10 & 20 \\
\hline \multirow[t]{2}{*}{4} & & 25 \\
\hline & & 30 \\
\hline Temperatura $\left({ }^{\circ} \mathrm{C}\right)$ & Pressão (bars) & Densidade $(\mathrm{g} / \mathrm{mL})$ \\
\hline 40 & 200 & 0,84 \\
\hline 40 & 250 & 0,88 \\
\hline 40 & 318 & 0,92 \\
\hline 50 & 110 & 0,50 \\
\hline 50 & 170 & 0,74 \\
\hline 50 & 197 & 0,78 \\
\hline 50 & 234 & 0,82 \\
\hline 50 & 284 & 0,86 \\
\hline 60 & 149 & 0,60 \\
\hline 60 & 169 & 0,66 \\
\hline 60 & 187 & 0,70 \\
\hline 60 & 244 & 0,78 \\
\hline 60 & 287 & 0,82 \\
\hline
\end{tabular}

1 Solventes para reconstituição da amostra: hexano, clorofórmio, acetona, acetonitrila e metanol.

2 Solvente para reconstituição da amostra: hexano. 
TABELA 2. Condições otimizadas de extração supercrítica estabelecidas para tocoferóis.

\begin{tabular}{lcc}
\hline Condições de extração & Pré-extração & Extração \\
\hline Temperatura da câmara de extração & $80^{\circ} \mathrm{C}$ & $50^{\circ} \mathrm{C}$ \\
Pressão & 76 bars & $197 \mathrm{bars}$ \\
Densidade & $0,15 \mathrm{~g} / \mathrm{mL}$ & $0,78 \mathrm{~g} / \mathrm{mL}$ \\
Fluxo do $\mathrm{CO}_{2}$-SC & $3,0 \mathrm{~mL} / \mathrm{min}$ & $3,0 \mathrm{~mL} / \mathrm{min}$ \\
Tempo de extração (dinâmica) & $8 \mathrm{~min}$ & $25 \mathrm{~min}$ \\
Temperatura do restritor & $55^{\circ} \mathrm{C}$ & $55^{\circ} \mathrm{C}$ \\
Temperatura do coletor & $20^{\circ} \mathrm{C}$ & $20^{\circ} \mathrm{C}$ \\
\hline Condições de reconstituição & & \\
\hline Solvente de lavagem do coletor & Acetonitrila & $\mathrm{Hexano}$ \\
Volume do solvente & $1,8 \mathrm{~mL}$ & $1,8 \mathrm{~mL}$ \\
Fluxo do solvente & $2,0 \mathrm{~mL} / \mathrm{min}$ & $2,0 \mathrm{~mL} / \mathrm{min}$ \\
Temperatura do coletor & $20^{\circ} \mathrm{C}$ & $60^{\circ} \mathrm{C}$ \\
\hline
\end{tabular}

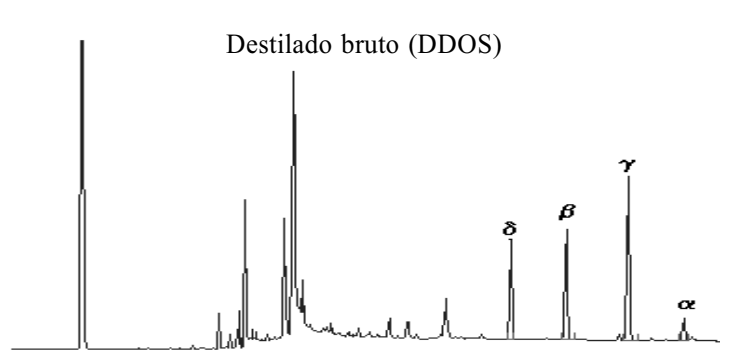

Extração com fluido supercrítico (EFS)

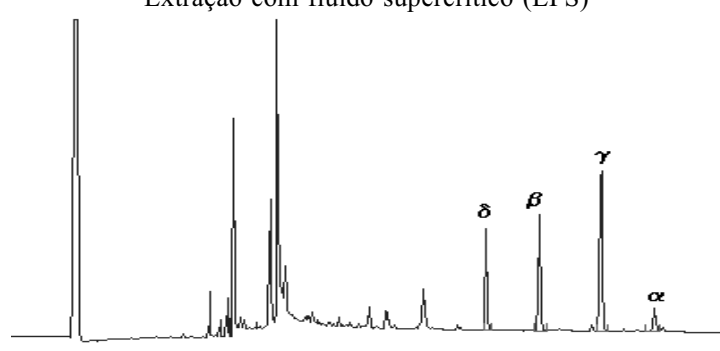

Extração com fluido supercrítico: pré-extração e extração

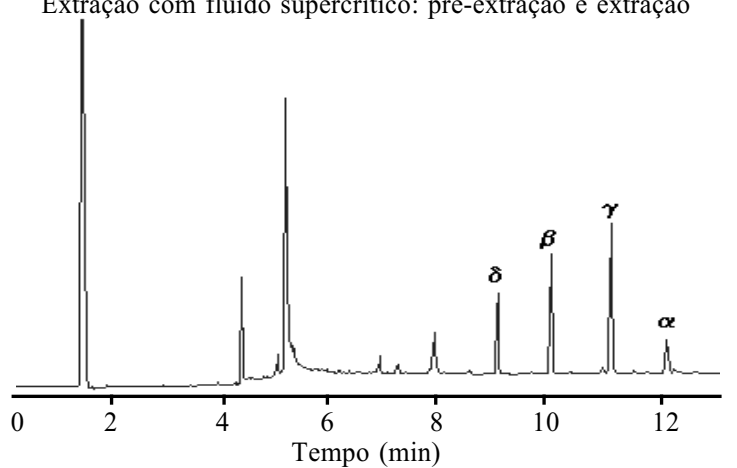

FIG. 1. Cromatogramas para a avaliação da extração supercrítica na obtenção do concentrado de tocoferóis a partir do destilado desodorizado do óleo de soja (DDOS). supercrítico, o que possibilitou o controle da capacidade de solvatação do solvente para o composto de interesse, permitindo a otimização da extração e, ou, a seletividade de uma classe de composto presente na amostra (Li et al., 1994). A variação da pressão comportou-se da mesma forma em todas as temperaturas. Sob condições isotérmicas, a densidade e a capacidade de solvatação do $\mathrm{CO}_{2}$-SC aumentaram com a elevação da pressão, até atingir seu valor máximo. A variável densidade apresentou-se significante apenas quanto à temperatura de $50^{\circ} \mathrm{C}$, obtendo o ótimo a $0,78 \mathrm{~g} / \mathrm{mL}$ (197 bars). Em razão do alto coeficiente de difusão do fluido supercrítico, o tempo de extração, dependendo da complexidade da amostra e da solubilidade relativa dos respectivos compostos de interesse (Levy et al., 1989), varia de 2 a 45 minutos. Inicialmente, utilizou-se a extração estática de 0,5 e 10 minutos, seguida por 20 minutos de extração dinâmica à temperatura de $50^{\circ} \mathrm{C}$ e pressão de 197 bars $(0,78 \mathrm{~g} / \mathrm{mL})$. A extração estática trata-se de uma diluição em que a amostra permanece por determinado tempo embebida no fluido supercrítico. Geralmente, é utilizada quando não se consegue uma separação rápida e completa do composto de interesse. Esta variável não apresentou significância quanto à extração dos tocoferóis.

Durante a extração dinâmica, dois processos ocorrem simultaneamente: transporte do componente extraído, e coleta desse composto após a expansão do $\mathrm{CO}_{2}$. Os tempos de extração dinâmica avaliados foram 15, 20, 25 e 30 minutos. Melhor rendimento de extração foi obtido no tempo de 25 minutos. 
Antes da primeira extração, o extrator supercrítico foi checado para assegurar que os tocoferóis foram quantitativamente extraídos e retidos no coletor. Isso foi realizado extraindo-se $50 \mu \mathrm{L}$ da solução-padrão de tocoferóis adsorvidos em papel-filtro. $\mathrm{O}$ extrato foi lavado do coletor com $1,8 \mathrm{~mL}$ de hexano, e analisado por cromatografia de fase gasosa. A mesma solução-padrão adicionada de $1,8 \mathrm{~mL}$ de hexano foi injetada no cromatógrafo de fase gasosa. Através da comparação das áreas dos picos foi possível observar uma recuperação de $100 \%$ dos tocoferóis pelo método de extração otimizado.

\section{CONCLUSÕES}

1. É possível utilizar o dióxido de carbono supercrítico na extração de tocoferóis.

2. A combinação de duas extrações, em que variam densidade, temperatura e tempo de extração, eleva a concentração de tocoferóis totais do destilado desodorizado do óleo de soja; a pré-extração, em baixa pressão, ocasiona a remoção de interferentes.

\section{REFERÊNCIAS}

GONÇALVES, M.; VASCONCELOS, A.M.P.; GOMES, A. Application of supercritical fluid extraction to the deacidification of olives oils. Journal of the American Oil Chemists' Society, Champaign, v.68, p.474-480, 1991.

HICKMAN, K.C.D. Commercial molecular distillation. Industrial and Engineering Chemistry, Washington, DC, v.39, p.686-694, 1947.

KARRER, P.V.; FRITSZSHE, B.H.; RINGIER, B.H $\alpha$-tocopherol. Helvetica Chemical Acta, Washington, DC, v.21, p.520-521, 1938.

LEE, H.; CHUNG, B.H.; PARK, Y.H. Concentration of tocopherols from soybean sludge by supercritical carbon dioxide. Journal of the American Oil
Chemists' Society, Champaign, v.68, p.571-573, 1991.

LEVY, J.M.; CAVALIER, R.A.; BOSCH, T.N.; RYNASKI, A.F.; HUHAK, W.E. Multidimensional supercritical fluid chromatography and supercritical fluid extraction. Journal of Chromatographic Science, Niles, IL, v.27, p.341-346, 1989.

LI, K.; ONG, C.P.; LI, S.F.Y. Systematic multivariate optimization of supercritical fluid extraction. Journal of Chromatographic Science, Niles, IL, v.32, p.53-56, 1994.

MARKS, C. Determination of free tocopherols in deodorizer distillate by capillary gas chromatography. Journal of the American Oil Chemists' Society, Champaign, v.65, p.1936-1939, 1988.

NICOLINO, A.P.N. Utilização do $\mathbf{C O}_{2}$ supercrítico para concentração de tocoferóis do destilado desodorizado do óleo de soja. Viçosa, MG : UFV, Impr. Univ., 1995. 64p. Tese de Mestrado.

QUAIFE, M.L.; HARRIS, P.L. Molecular distillation as a step in the chemical determination of total and gamma tocopherols. Industrial and Engineering Chemistry, Washington, DC, v.18, p.707-709, 1946.

SAITO, M.; YAMAUCHI, Y.; INOMATA, K.; KOTTKAM, P.W. Enrichment of tocopherols in wheat germ by directly coupled supercritical fluid extraction with semipreparative supercritical fluid chromatography. Journal of Chromatographic Science, Niles, IL, v.27, p.79-85, 1989.

SLOVER, H.T.; THOMPSON, R.H.; MEROLA, G.V. Determination of tocopherols and sterols by capillary gas chromatography. Journal of the American Oil Chemists' Society, Champaign, v.60, p.1524-1528, 1983.

WOERFEL, J.B. Processing and utilization of by-products from soy oil processing. Journal of the American Oil Chemists' Society, Champaign, v.58, p.188191, 1981. 